\title{
Prognostic significance of serum tumor marker normalization in the perioperative period for patients with advanced gastric cancer
}

\author{
Can $\mathrm{Hu}^{1,2 \#}$, Yanqiang Zhang ${ }^{2,3 \#}$, Jingli Xu ${ }^{1}$, Wujie Chen ${ }^{2}$, Pengcheng $\mathrm{Yu}^{1}$, Yi Wang ${ }^{1}$, Zhehan Bao ${ }^{1}$, \\ Ruolan Zhang ${ }^{1}$, Zhiyuan $\mathrm{Xu}^{2,3}$, Xiangdong Cheng ${ }^{2,3}$ \\ ${ }^{1}$ Second Clinical Medical College, Zhejiang Chinese Medical University, Hangzhou, China; ${ }^{2}$ Department of Gastric Surgery, the Cancer Hospital of \\ the University of Chinese Academy of Sciences (Zhejiang Cancer Hospital), Institutes of Basic Medicine and Cancer (IBMC), Chinese Academy of \\ Sciences, Hangzhou, China; ${ }^{3}$ Diagnosis and Therapy Center of Upper Gastrointestinal Tumor in Zhejiang Province, Hangzhou, China \\ Contributions: (I) Conception and design: Z Xu, X Cheng; (II) Administrative support: X Cheng; (III) Provision of study materials or patients: C Hu, \\ Y Zhang; (IV) Collection and assembly of data: C Hu, Y Zhang, J Xu, W Chen, P Yu, Y Wang, Z Bao, R Zhang; (V) Data analysis and interpretation: \\ C Hu, Y Zhang; (VI) Manuscript writing: All authors; (VII) Final approval of manuscript: All authors. \\ \#These authors contributed equally to this work. \\ Correspondence to: Xiangdong Cheng. The Cancer Hospital of the University of Chinese Academy of Sciences (Zhejiang Cancer Hospital), Institutes \\ of Basic Medicine and Cancer (IBMC), Chinese Academy of Sciences, Hangzhou 310022, China. Email: chengxd@zjcc.org.cn; Zhiyuan Xu. The \\ Cancer Hospital of the University of Chinese Academy of Sciences (Zhejiang Cancer Hospital), Institutes of Basic Medicine and Cancer (IBMC), \\ Chinese Academy of Sciences, Hangzhou 310022, China. Email: getfar@foxmail.com.
}

Background: Neoadjuvant chemotherapy (NCT) was developed to improve the prognosis of patients with advanced gastric cancer (AGC). Some studies have confirmed the diagnostic and prognostic value of various serum tumor markers in gastric cancer. However, most of these studies were focused on the value of preoperative and postoperative tumor markers in patients undergoing surgery with or without adjuvant therapy, and only a few studies focused on AGC patients undergoing NCT.

Methods: We retrospectively analyzed the data of consecutive patients with histologically confirmed AGC who received NCT prior to surgical resection at Zhejiang Cancer Hospital from January 2010 to September 2018. The prognostic impact of tumor markers before and after NCT, including Carcinoembryonic antigen (CEA), Carbohydrate antigen199 (CA199), Carbohydrate antigen125 (CA125), Alpha-FetoProtein (AFP), Carbohydrate antigen242 (CA242), and Carbohydrate antigen724 (CA724), were evaluated using KaplanMeier log-rank survival analysis. The association between tumor marker normalization during preoperative chemotherapy and clinicopathological characteristics was also investigated.

Results: Four hundred and seventy-two patients were included in the study. The levels of CEA, CA199, CA125, CA242, and CA724 before NCT could predict prognosis, and the levels of CA199, CA125, CA242, and CA724 after NCT were correlated with prognosis. The overall survival (OS) rate decreased with an increasing number of positive tumor markers before and after preoperative chemotherapy. Tumor marker abnormalization after NCT was not related to chemotherapy, whereas patients with tumor marker normalization after NCT obtained survival benefits.

Conclusions: Tumor markers before and after NCT, such as CA199, CA125, CA242, and CA724, have a discriminatory ability for patients with GC. The normalization of tumor markers after NCT was associated with better survival.

Keywords: Tumor marker; neoadjuvant chemotherapy (NCT); gastric cancer

Submitted Dec 21, 2021. Accepted for publication Jan 27, 2022.

doi: $10.21037 /$ atm-22-109

View this article at: https://dx.doi.org/10.21037/atm-22-109 


\section{Introduction}

Gastric cancer (GC) is the fifth most common cancer and the third most common cause of cancer death globally (1). Surgery is the best treatment for GC patients. However, the disease is often asymptomatic in the early stage and is usually diagnosed with invasion or metastasis in an advanced stage, at which point the opportunity for surgery is already lost (2). In recent years, some studies have shown that the neoadjuvant chemotherapy (NCT) treatment has significantly improved the prognosis of patients with advanced gastric cancer (AGC). And according to NCCN Guidelines and CSCO Guideline, the GC patients in locally advanced and/or lymph node metastasis are recommended to NCT treatment before surgery (3-5). However, due to the high heterogeneity in AGC, the survival of AGC patients with the same stage differs. Therefore, identifying effective tumor markers to evaluate the prognosis and survival of GC patients is crucial.

At present, carcinoembryonic antigen (CEA), carbohydrate antigen (CA)-199, CA-125, CA-242, CA724 , and alpha fetoprotein (AFP) are the common serum markers for early diagnosis and monitoring of cancer. A number of studies have confirmed that some serum markers play an important role in predicting the prognosis and monitoring of GC $(6,7)$. However, most previous studies mainly focused on the clinical prognostic significance of preoperative and postoperative levels of serum tumor markers (8-10). Presently, there are few studies evaluating the prognostic value of serum tumor marker normalization in the perioperative period for GC patients receiving NCT.

Therefore, the aim of this study was to determine the value of the changes of serum markers in patients with GC before and after NCT. Moreover, we also sought to determine whether patients with positive tumor markers before NCT that normalized after NCT had a better survival than patients who still had positive tumor markers.

We present the following article in accordance with the REMARK reporting checklist (available at https://atm. amegroups.com/article/view/10.21037/atm-22-109/rc).

\section{Methods}

\section{Selection criteria and patients}

We retrospectively analyzed the data of consecutive patients with histologically confirmed GC/Esophagogastric junction cancer (EGJC) who received NCT prior to surgical resection at Zhejiang Cancer Hospital from January 2010 to September 2018. The inclusion criterion was as follows: (I) pT2-4NxM0 resectable GC; (II) GC/EGJC confirmed by pathological examination; (III) patients with D2 lymphadenectomy; (IV) patients with negative resection margins; and $(\mathrm{V})$ patients with complete clinical data. The exclusion criteria were as follows: (I) metastatic GC; and (II) patients with incomplete medical records. Finally, 472 patients were included in the study.

The medical records of all identified patients were reviewed. Data including demographic characteristics, tumor characteristics, treatment regimens, treatment responses, and survival were collected. This study was approved by the ethics committee of the Cancer Hospital of the University of Chinese Academy of Sciences (Zhejiang Cancer Hospital) (No. IRB-2020-300), and conformed to the tenets of the Declaration of Helsinki (as revised in 2013). Informed consent from the patients was waived because of the retrospective nature of this study.

\section{Detection of tumor markers}

Serum CEA, CA199, CA125, AFP, CA242, and CA724 concentrations were recorded from routine clinical testing. Tumor markers before NCT were measured when the patient was first diagnosed with GC, prior to receiving any treatment. Tumor markers after NCT were measured before the patients received surgery. According to the routine clinical testing, the recommended upper cut-off values for CEA, CA199, CA125, AFP, CA242, and CA724 were $5 \mathrm{ng} / \mathrm{mL}, 37 \mathrm{U} / \mathrm{mL}, 35 \mathrm{U} / \mathrm{mL}, 8.1 \mathrm{ng} / \mathrm{mL}, 20 \mathrm{U} / \mathrm{mL}$, and $6.9 \mathrm{U} / \mathrm{mL}$, respectively. Testing values over the cut-offs were regarded as positive. Additionally, the normalization of tumor markers was defined as the change from the abnormal tumor marker group before NCT to the normal tumor marker group after NCT.

\section{Clinicopathological characteristics}

The following clinicopathological characteristics were collected: age, sex, NCT regimens, tumor location, tumor size, Lauren classification, degree of differentiation, nerve invasion, vessel invasion, neoadjuvant pathologic $\mathrm{T}$ (ypT) stage, neoadjuvant pathologic $\mathrm{N}$ (ypN) stage, and neoadjuvant pathologic TNM (ypTNM) stage via the pathological results according to the Union for international Cancer Control (UICC) guidelines ( $8^{\text {th }}$ edition). 


\section{Assessment and follow-up}

After two cycles of NCT treatment, clinical efficacy was assessed based on computed tomography (CT) or magnetic resonance imaging (MRI). After systemic treatment, the patients were regularly examined every 3 months for the first year and every 6 months thereafter. The final follow-up assessment was conducted in September 2021. Most routine follow-up appointments included a physical examination, laboratory testing (including serum tumor marker and abdominal computed tomography), and an annual endoscopic examination.

\section{Statistical analysis}

Statistical analysis was performed using IBM SPSS Statistics for Mac OS, version 20.0 (IBM Corp., Armonk, NY, USA). Categorical variables were compared using the $\chi^{2}$ test or Fisher's exact test, while differences in continuous variables between groups were analyzed using the Mann-Whitney U test. Survival curves were estimated using the Kaplan-Meier method. Multivariate analyses were performed using a Cox stepwise proportional hazard model. A P value of $<0.05$ was considered statistically significant.

\section{Results}

\section{Clinicopathological characteristics and clinical results}

In total, 472 patients with histologically confirmed GC/ EGJC who received NCT prior to surgical resection at Zhejiang Cancer Hospital were enrolled in this study. As shown in Table 1, among these 472 patients, the median age was 61 years (range, 21-80 years), and there were $349(73.94 \%)$ males and 123 females (26.06\%). Also, 223 $(47.25 \%)$ tumors were located in lower third part of the stomach, while 134 (28.39\%) tumors were located in upper third part of the stomach. $430(91.10 \%)$ patients received NCT with a two-drug regimen, while $36(8.90 \%)$ patients re-received NCT with a three-drug regimen.

Pathologically, 15 (3.18\%) were defined as pathological complete response (pCR), while $72(15.25 \%)$ patients were in the pT1-2 stage, and 384 (81.57\%) patients were in pT34 stage. Furthermore, 319 (67.58\%) patients had lymph node metastasis, while signet-ring cell were found in 85 (18.01\%) patients. Up to now, 252 patients had died and 220 patients were still alive. The median follow-up time of all patients was 39 months (range, 1-137 months), and the 3 -year OS was $54.45 \%$.

\section{Survival rates based on tumor markers before and after preoperative markers}

Due to the retrospective nature of this study, the data of pre-serum tumor markers before NCT of 92 patients who received NCT in another hospital were unavailable. The levels of serum tumor markers before NCT were measured within 1 week prior to NCT, and the serum levels after NCT were measured within 1 week before gastrectomy.

The results showed that for the level of CA199, CA125, CA242, and CA742 before or after NCT, all positive groups had a worse prognosis (all $\mathrm{P}<0.05$ ), but there was no significant difference between AFP-positive group and AFP-negative group before or after NCT (as shown in Figure 1). Interestingly, no significant difference was observed between the CEA-positive and CEA-negative groups after NCT, while patients with positive CEA before preoperative chemotherapy had a worse prognosis $(\mathrm{P}=0.0227)$ (as shown in Figure 2).

\section{Normalization of the level of serum tumor markers during preoperative therapy and survival}

Pre-treatment, there were 136 patients with no positive tumor markers, while 244 patients had positive tumor markers, including 218 patients with one, two, or three positive tumor markers and 26 patients with more than three positive tumor markers. After preoperative chemotherapy, there were 189 patients with no positive tumor markers, while 283 patients had positive tumor markers, including 269 patients with one, two, or three positive tumor markers and 14 patients with more than three positive tumor markers.

The Kaplan-Meier curves for the 3-year OS rate decreased with an increasing number of positive tumor markers before and after preoperative chemotherapy. Among 244 patients with positive tumor markers before pre-treatment, the status of tumor markers in 68 patients returned to normal. Also, the 3-year OS of patients with tumor markers normalized after preoperative chemotherapy was similar to those patients with negative tumor markers before pre-treatment (as shown in Figure 3). Upon further analysis, patients with normalized CA199, CA125, or CA724 had a similar 3-year OS to those patients with negative CA199, CA125, or CA724 before pre-treatment. However, this phenomenon was not observed the CEA and CA242 groups (as shown in Figure 4).

Moreover, the association between tumor marker 
Table 1 Patient characteristics

\begin{tabular}{|c|c|}
\hline Variable & $\mathrm{N}(\%)$ \\
\hline \multicolumn{2}{|l|}{ Age (years) } \\
\hline$>60$ & $253(53.60)$ \\
\hline$\leq 60$ & $219(86.56)$ \\
\hline \multicolumn{2}{|l|}{ Sex } \\
\hline Male & $349(73.94)$ \\
\hline Female & $123(26.06)$ \\
\hline \multicolumn{2}{|l|}{ урT stage } \\
\hline 0 & $15(3.18)$ \\
\hline $1-2$ & $72(15.25)$ \\
\hline $3-4$ & $385(81.57)$ \\
\hline \multicolumn{2}{|l|}{ ypN stage } \\
\hline 0 & $153(32.42)$ \\
\hline 1 & $93(19.70)$ \\
\hline 2 & $106(22.46)$ \\
\hline 3 & $120(25.42)$ \\
\hline \multicolumn{2}{|l|}{ Tumor location } \\
\hline Upper third & $134(28.39)$ \\
\hline Middle third & $115(24.36)$ \\
\hline Lower third & $223(47.25)$ \\
\hline \multicolumn{2}{|l|}{ Differentiated degree } \\
\hline Well & $7(1.48)$ \\
\hline Moderate & $67(14.19)$ \\
\hline Low & $398(84.99)$ \\
\hline \multicolumn{2}{|c|}{ Signet-ring cell component } \\
\hline Yes & $85(18.01)$ \\
\hline No & 387 (81.99) \\
\hline \multicolumn{2}{|c|}{ No. of cycles of preoperative chemotherapy } \\
\hline$\leq 4$ & $436(92.37)$ \\
\hline$>4$ & $36(7.36)$ \\
\hline \multicolumn{2}{|c|}{ Chemotherapeutic regimens } \\
\hline Two-drug regimen & $430(91.10)$ \\
\hline Three-drug regimen & $42(8.90)$ \\
\hline \multicolumn{2}{|l|}{ CEA-pre } \\
\hline Positive & $118(25.00)$ \\
\hline Negative & $262(55.51)$ \\
\hline Unknown & $92(19.49)$ \\
\hline
\end{tabular}

Table 1 (continued)
Table 1 (continued)

\begin{tabular}{|c|c|}
\hline Variable & N (\%) \\
\hline \multicolumn{2}{|l|}{ CA199-pre } \\
\hline Positive & $120(25.42)$ \\
\hline Negative & $260(55.08)$ \\
\hline Unknown & $92(19.49)$ \\
\hline \multicolumn{2}{|l|}{ CA125-pre } \\
\hline Positive & 44 (9.32) \\
\hline Negative & $226(47.88)$ \\
\hline Unknown & 92 (19.49) \\
\hline \multicolumn{2}{|l|}{ AFP-pre } \\
\hline Positive & 38 (8.05) \\
\hline Negative & $342(72.46)$ \\
\hline Unknown & 92 (19.49) \\
\hline \multicolumn{2}{|l|}{ CA242-pre } \\
\hline Positive & $63(13.35)$ \\
\hline Negative & $317(67.16)$ \\
\hline Unknown & $92(19.49)$ \\
\hline \multicolumn{2}{|l|}{ CA724-pre } \\
\hline Positive & 92 (19.49) \\
\hline Negative & 288 (61.02) \\
\hline Unknown & 92 (19.49) \\
\hline \multicolumn{2}{|l|}{ CEA-pos } \\
\hline Positive & $124(26.27)$ \\
\hline Negative & $348(73.73)$ \\
\hline \multicolumn{2}{|l|}{ CA199-pos } \\
\hline Positive & $86(18.22)$ \\
\hline Negative & $386(81.78)$ \\
\hline \multicolumn{2}{|l|}{ CA125-pos } \\
\hline Positive & $30(6.36)$ \\
\hline Negative & $442(93.64)$ \\
\hline \multicolumn{2}{|l|}{ AFP-pos } \\
\hline Positive & 91 (19.28) \\
\hline Negative & 381 (80.72) \\
\hline \multicolumn{2}{|l|}{ CA242-pos } \\
\hline Positive & $38(8.05)$ \\
\hline Negative & 434 (91.95) \\
\hline \multicolumn{2}{|l|}{ CA724-pos } \\
\hline Positive & $113(23.94)$ \\
\hline Negative & 359 (76.06) \\
\hline
\end{tabular}



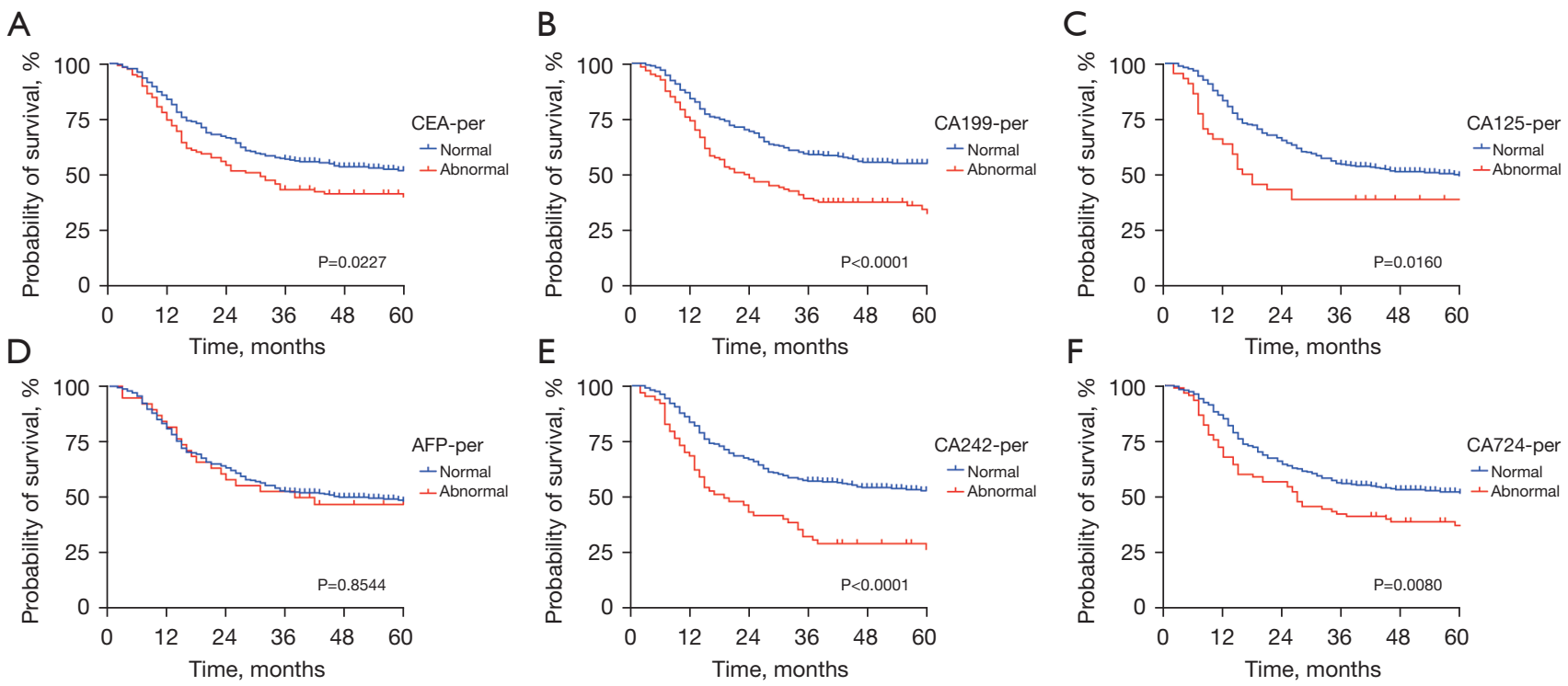

Figure 1 Kaplan-Meier analysis of the OS of AGC patients stratified by CEA (A), CA199 (B), CA125 (C), AFP (D), CA242 (E), and CA724 (F) before NCT. AGC, advanced gastric cancer; CEA, carcinoembryonic antigen; AFP, alpha fetoprotein; NCT, neoadjuvant chemotherapy.
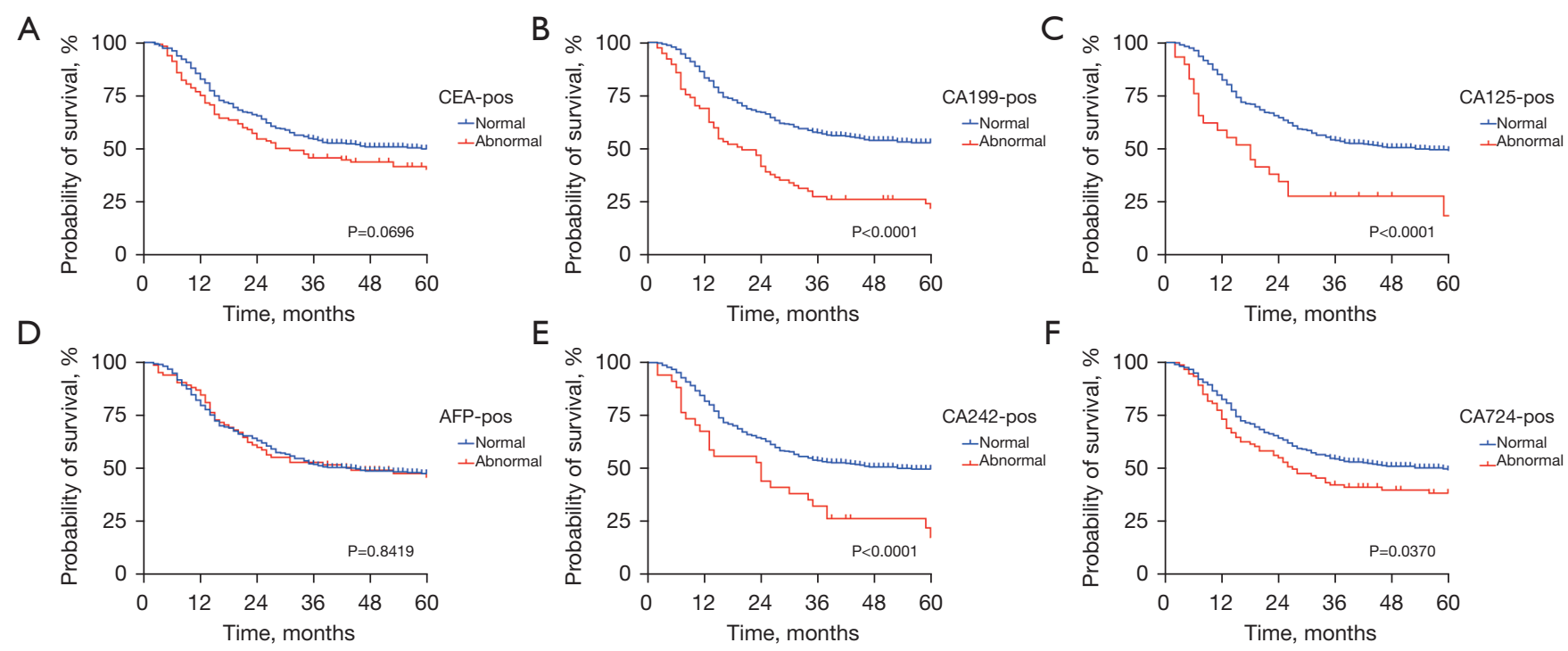

Figure 2 Kaplan-Meier analysis of the OS of AGC patients stratified by CEA (A), CA199 (B), CA125 (C), AFP (D), CA242 (E), and CA724 (F) after NCT. AGC, advanced gastric cancer; CEA, carcinoembryonic antigen; AFP, alpha fetoprotein; NCT, neoadjuvant chemotherapy.

normalization during preoperative chemotherapy and clinicopathological characteristics were confirmed (as shown in Table 2). The results also showed a significant correlation between tumor marker normalization and the number of positive tumor markers after surgery, whereas tumor marker normalization was not significantly associated with sex, age, ypT stage, ypN stage, ypTNM stage, tumor location, differentiated degree, and signet-ring cell component.

According to the univariate analysis (Table 3), the significant prognostic factors for survival in patients with positive tumor markers before NCT were ypT stage, ypN stage, ypTNM stage, differentiated degree, signet-ring cell component, level of tumor markers (CEA, CA199, CA125, CA242, and CA724) before NCT, number of positive 
A

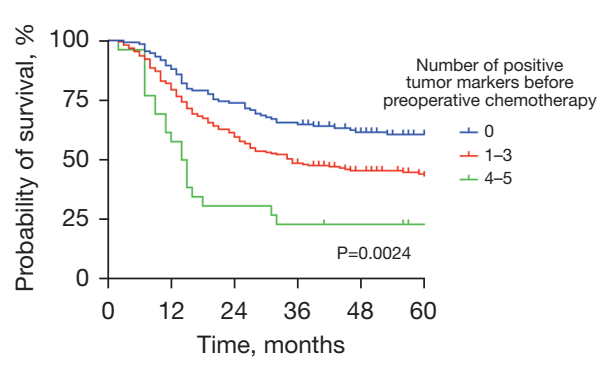

C

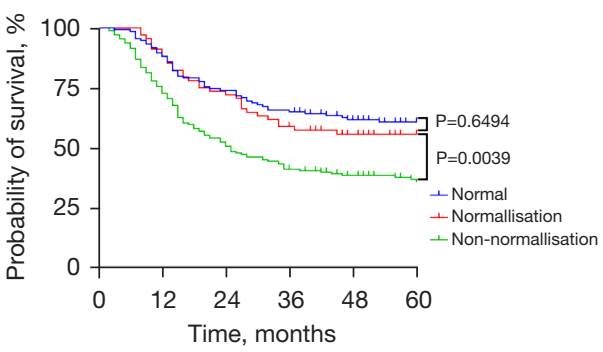

B

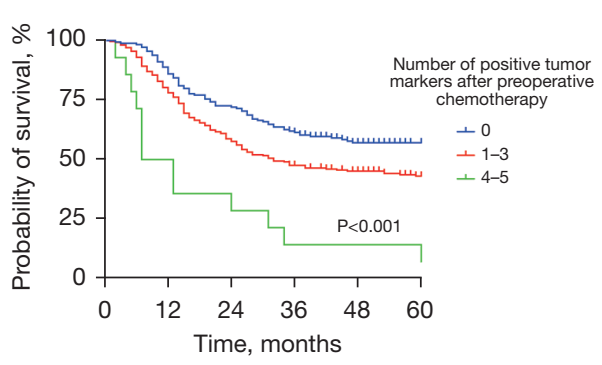

D

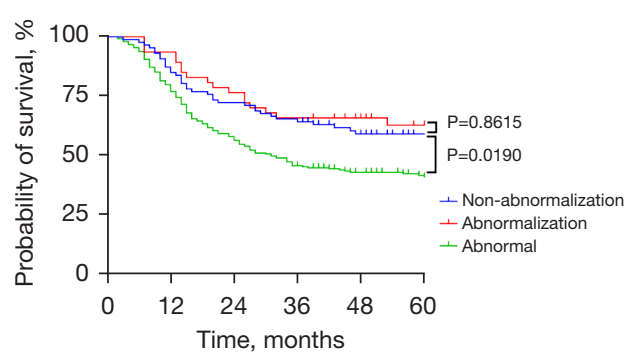

Figure 3 Kaplan-Meier analysis of the OS of AGC patients who received NCT. Association between the number of positive tumor markers before NCT (A) and after NCT (B) with OS of all patients who received NCT. (C) Association between tumor marker normalization or non-normalization after NCT and the OS of all patients. (D) Association between the tumor marker abnormalization or nonabnormalization after NCT and the OS of all patients. AGC, advanced gastric cancer; NCT, neoadjuvant chemotherapy.

A
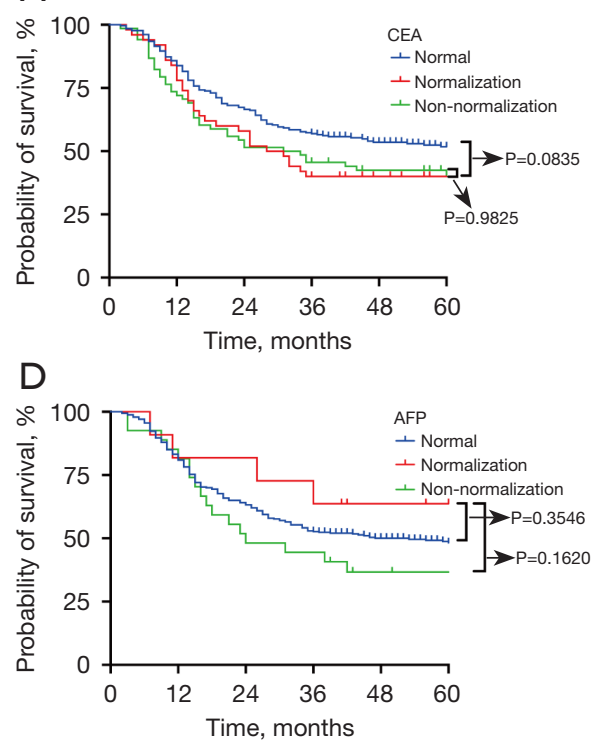

B

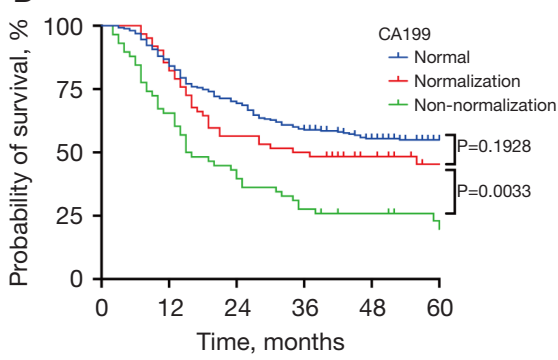

$\mathrm{E}$

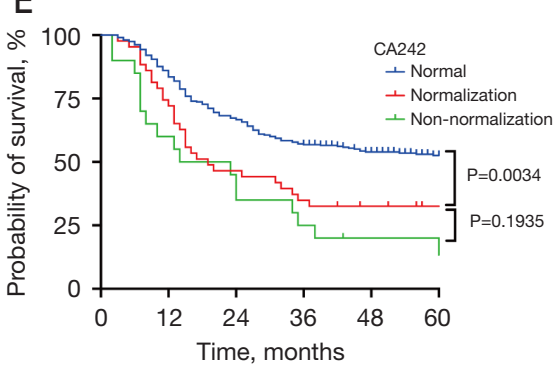

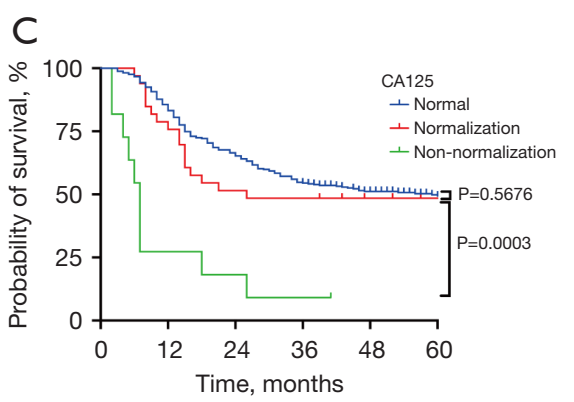

$\mathrm{F}$

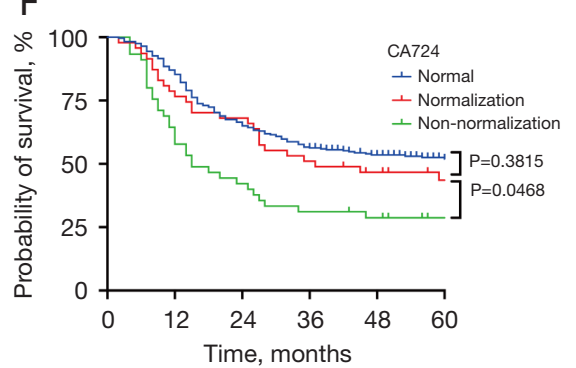

Figure 4 Kaplan-Meier analysis of the OS of AGC patients who received NCT. Association between CEA (A), CA199 (B), CA125 (C), AFP (D), CA242 (E), and CA724 (F) normalization or non-normalization after NCT and the OS of all patients. AGC, advanced gastric cancer; CEA, carcinoembryonic antigen; AFP, alpha fetoprotein; NCT, neoadjuvant chemotherapy. 
Table 2 Correlation between tumor marker normalization during preoperative chemotherapy and clinicopathological characteristics

\begin{tabular}{|c|c|c|c|c|}
\hline Variable & Normalization & $\begin{array}{c}\text { Non- } \\
\text { normalization }\end{array}$ & $\chi^{2}$ & $P$ \\
\hline Age (years) & & & 2.070 & 0.150 \\
\hline$>60$ & 44 & 96 & & \\
\hline$\leq 60$ & 24 & 80 & & \\
\hline Sex & & & 1.293 & 0.255 \\
\hline Male & 56 & 133 & & \\
\hline Female & 12 & 43 & & \\
\hline урT stage & & & 0.670 & 0.413 \\
\hline $1-2$ & 8 & 28 & & \\
\hline $3-4$ & 60 & 148 & & \\
\hline ypN stage & & & 3.006 & 0.083 \\
\hline 0 & 25 & 45 & & \\
\hline $1+2+3$ & 43 & 131 & & \\
\hline yTNM stage & & & 0.844 & 0.358 \\
\hline$I+I I$ & 23 & 49 & & \\
\hline III & 45 & 127 & & \\
\hline Tumor location & & & 0.757 & 0.685 \\
\hline Upper third & 20 & 53 & & \\
\hline Middle third & 12 & 39 & & \\
\hline Lower third & 36 & 84 & & \\
\hline Differentiated d & & & 0.635 & 0.728 \\
\hline Well & 1 & 3 & & \\
\hline Moderate & 12 & 24 & & \\
\hline Low & 55 & 149 & & \\
\hline Signet-ring cell & nponent & & 1.591 & 0.222 \\
\hline Yes & 7 & 29 & & \\
\hline No & 61 & 147 & & \\
\hline \multicolumn{3}{|c|}{ Number of positive tumor markers before NCT } & 5.893 & $0.015^{\star}$ \\
\hline $1-3$ & 66 & 152 & & \\
\hline $4-5$ & 2 & 24 & & \\
\hline
\end{tabular}

*, $\mathrm{P}<0.05$. NCT, neoadjuvant chemotherapy.

tumor markers before NCT, and normalization of tumor marker after NCT. The Cox proportional hazard model was used for multivariate analysis, and the results showed that ypT stage, ypN stage, ypTNM stage, differentiated degree, signet-ring cell component, level of tumor markers (CEA, CA199, CA125, and CA242) before NCT, number of positive tumor markers before NCT, and normalization of tumor marker after NCT were independent risk factors.

\section{Discussion}

Being the third leading cause of cancer-related death world, GC diagnosis and treatment is a significant concern. Most Chinese GC patients are already at advanced stage at the time of diagnosis. Surgery is the main treatment for GC, but the long-term survival rate of patients with AGC after surgery is still less than 20-30\%. Recently, the RESOLVE clinical trial showed that NCT treatment with oxaliplatin plus S-1 have a meaningful improvement compared with adjuvant chemotherapy in patients with locally AGC. Therefore, NCT was developed to improve the prognosis of patients with AGC. The advantages of NCT, including reducing tumor size, improving the rate of R0 resection, and improving OS and DFS, have been demonstrated in numerous large studies. However, due to the heterogeneity of GC, the clinical response rate of NCT is barely satisfactory. Therefore, there is a pressing need to mine reliable indicators that can predict chemotherapy sensitivity, in order to screen out AGC patients that are suitable for NCT.

Serum tumor markers are widely used in early diagnosis, efficacy evaluation, and monitoring disease recurrence. Some studies have confirmed the clinical diagnostic and prognostic value of various serum tumor markers in GC. Wang et al. showed that CEA can be used to diagnose GC and improve the diagnostic sensitivity of imaging examination for lymph node involvement (9). CA199 is also considered to be a sensitive marker for liver and peritoneal recurrence and metastasis (11). However, most of these studies were focused on the value of preoperative and postoperative tumor markers in patients undergoing surgery with or without adjuvant therapy, and studies focused on AGC patients undergoing NCT are rare.

In our study, the levels of CEA, CA199, CA125, CA242, and CA724 before NCT could predict prognosis, and the level of CA199, CA125, CA242, and CA724 after NCT were correlated with prognosis. A combination of multiple tumor markers improves the prediction of survival compared to a single tumor marker (12). Therefore, all patients were grouped according to the number of positive tumor markers, and the results showed that the 3-year OS rate decreased with an increasing number of positive 
Table 3 Prognostic factors in univariate and multivariate analyses for patients with positive tumor markers

\begin{tabular}{|c|c|c|c|c|}
\hline Characteristics & $\mathrm{P}$ (univariate) & HR (multivariate) & 95\% Cl (multivariate) & $\mathrm{P}$ (multivariate) \\
\hline Age & 0.174 & - & - & - \\
\hline ypT stage & $\mathrm{P}<0.001^{*}$ & 0.378 & $0.305-0.470$ & $\mathrm{P}<0.001^{*}$ \\
\hline ypN stage & $\mathrm{P}<0.001^{*}$ & 4.869 & $4.135-5.734$ & $P<0.001^{*}$ \\
\hline Tumor location & 0.963 & - & - & - \\
\hline Differentiated degree & $\mathrm{P}<0.001^{*}$ & 0.481 & $0.416-0.558$ & $\mathrm{P}<0.001^{*}$ \\
\hline Signet-ring cell component & $\mathrm{P}<0.001^{*}$ & 0.866 & $0.774-0.970$ & $0.013^{*}$ \\
\hline CEA-pre & $0.002^{*}$ & 0.872 & $0.790-0.964$ & $0.007^{\star}$ \\
\hline AFP-pre & 0.520 & - & - & - \\
\hline CA242-pre & $P<0.001^{*}$ & 1.181 & $1.053-1.325$ & $0.005^{*}$ \\
\hline CA724-pre & $0.002^{*}$ & 0.954 & $0.863-1.055$ & 0.359 \\
\hline Number of positive tumor markers before NCT & $\mathrm{P}<0.001^{*}$ & 1.555 & $1.312-1.844$ & $\mathrm{P}<0.001^{*}$ \\
\hline Normalization of tumor markers after NCT & $\mathrm{P}<0.001^{*}$ & 1.375 & $1.240-1.525$ & $\mathrm{P}<0.001^{*}$ \\
\hline
\end{tabular}

*, $\mathrm{P}<0.05$. NCT, neoadjuvant chemotherapy; HR, hazard ratio; $\mathrm{Cl}$, confidence interval.

tumor markers before and after preoperative chemotherapy. These results indicated that the high level of tumor markers both before and after NCT may be associated with poor prognosis in AGC.

Furthermore, we divided all of the patients into a normal group and an abnormal group according to the status of tumor markers before NCT. For patients with normal tumor markers before NCT, $34.56 \%$ patients had positive tumor markers after NCT, and the abnormalization of tumor markers after NCT was not associated with OS for these patients. In addition to tumor progression, abnormalization of tumor markers after NCT was not related to chemotherapy. For patients with abnormal tumor markers before NCT, $27.87 \%$ patients had normal tumor markers after NCT, and the prognosis of patients with normalized tumor markers was significantly better than that of patients with non-normalized after NCT, and there was no significant difference in 3-year OS between patients with normalized tumor markers after NCT and those with normal tumor markers before NCT. Also, the normalization of tumor markers after NCT was an independent risk factor for patients with positive tumor markers before NCT. Further analysis showed a significant correlation between tumor marker normalization and the number of positive tumor markers after surgery, while tumor markers normalization was not significantly associated with sex, age, ypT stage, ypN stage, ypTNM stage, tumor location, differentiated degree, or signet-ring cell component. These results highlight that some patients with positive tumor markers may achieve obvious survival benefits from NCT treatment, especially for patients with $1-3$ positive tumor markers.

However, this study has several limitations that should be noted. Firstly, as a retrospective and single center study, there was possible selection bias and performance of analysis bias. For example, the pre-serum tumor marker before preoperative chemotherapy data in some patients who received preoperative chemotherapy in other hospitals were unavailable. Secondly, the significance of positive tumor markers in AGC needs to be further explored, and an effective prediction model for NCT efficacy and prognosis needs to be established. 


\section{Conclusions}

Tumor markers before and after NCT, such as CA199, CA125, CA242, and CA724, have a discriminatory ability for patients with GC. The normalization of tumor markers after NCT was associated with better survival.

\section{Acknowledgments}

Funding: This study was supported by the Diagnosis and Therapy Center of Upper Gastrointestinal Tumor (No. JBZX-202006); the Natural Science Foundation of Zhejiang Province (No. LY18H290006); the Key research and development project of Zhejiang Science and Technology Department (No. 2019C03049); the National Natural Science Foundation of China (Nos. 81973634, 82074245); and the Program of Zhejiang Provincial TCM Sci-tech Plan (Nos. 2019ZZ010, 2021ZA020).

\section{Footnote}

Reporting Checklist: The authors have completed the REMARK reporting checklist. Available at https://atm. amegroups.com/article/view/10.21037/atm-22-109/rc

Data Sharing Statement: Available at https://atm.amegroups. com/article/view/10.21037/atm-22-109/dss

Conflicts of Interest: All authors have completed the ICMJE uniform disclosure form (available at https://atm.amegroups. com/article/view/10.21037/atm-22-109/coif). All authors report funding from the Diagnosis and Therapy Center of Upper Gastrointestinal Tumor (No. JBZX-202006); the Natural Science Foundation of Zhejiang Province (No. LY18H290006); the Key research and development project of Zhejiang Science and Technology Department (No. 2019C03049); the National Natural Science Foundation of China (Nos. 81973634, 82074245); and the Program of Zhejiang Provincial TCM Sci-tech Plan (Nos. 2019ZZ010, 2021ZA020). The authors have no other conflicts of interest to declare.

Ethical Statement: The authors are accountable for all aspects of the work in ensuring that questions related to the accuracy or integrity of any part of the work are appropriately investigated and resolved. This study was approved by the ethics committee of the Cancer Hospital of the University of Chinese Academy of Sciences (Zhejiang
Cancer Hospital) (No. IRB-2020-300), and conformed to the tenets of the Declaration of Helsinki (as revised in 2013). Informed consent from the patients was waived because of the retrospective nature of this study.

Open Access Statement: This is an Open Access article distributed in accordance with the Creative Commons Attribution-NonCommercial-NoDerivs 4.0 International License (CC BY-NC-ND 4.0), which permits the noncommercial replication and distribution of the article with the strict proviso that no changes or edits are made and the original work is properly cited (including links to both the formal publication through the relevant DOI and the license). See: https://creativecommons.org/licenses/by-nc-nd/4.0/.

\section{References}

1. Smyth EC, Nilsson M, Grabsch HI, et al. Gastric cancer. Lancet 2020;396:635-48.

2. $\mathrm{Xu} \mathrm{Z,} \mathrm{Hu} \mathrm{C,} \mathrm{Yu} \mathrm{J,} \mathrm{et} \mathrm{al.} \mathrm{Efficacy} \mathrm{of} \mathrm{Conversion}$ Surgery Following Apatinib Plus Paclitaxel/S1 for Advanced Gastric Cancer With Unresectable Factors: A Multicenter, Single-Arm, Phase II Trial. Front Pharmacol 2021;12:642511.

3. Wang FH, Shen L, Li J, et al. The Chinese Society of Clinical Oncology (CSCO): clinical guidelines for the diagnosis and treatment of gastric cancer. Cancer Commun (Lond) 2019;39:10.

4. Cats A, Jansen EPM, van Grieken NCT, et al. Chemotherapy versus chemoradiotherapy after surgery and preoperative chemotherapy for resectable gastric cancer (CRITICS): an international, open-label, randomised phase 3 trial. Lancet Oncol 2018;19:616-28.

5. Ji Y, Du X, Zhu W, et al. Efficacy of Concurrent Chemoradiotherapy With S-1 vs Radiotherapy Alone for Older Patients With Esophageal Cancer: A Multicenter Randomized Phase 3 Clinical Trial. JAMA Oncol 2021;7:1459-66.

6. Feng F, Tian Y, Xu G, et al. Diagnostic and prognostic value of CEA, CA19-9, AFP and CA125 for early gastric cancer. BMC Cancer 2017;17:737.

7. Shimada H, Noie T, Ohashi M, et al. Clinical significance of serum tumor markers for gastric cancer: a systematic review of literature by the Task Force of the Japanese Gastric Cancer Association. Gastric Cancer 2014;17:26-33.

8. Song XH, Liu K, Yang SJ, et al. Prognostic Value of Changes in Preoperative and Postoperative Serum CA19- 
9 Levels in Gastric Cancer. Front Oncol 2020;10:1432.

9. Wang K, Jiang X, Ren Y, et al. The significance of preoperative serum carcinoembryonic antigen levels in the prediction of lymph node metastasis and prognosis in locally advanced gastric cancer: a retrospective analysis. BMC Gastroenterol 2020;20:100.

10. Lin JP, Lin JX, Ma YB, et al. Prognostic significance of pre- and post-operative tumour markers for patients with gastric cancer. Br J Cancer 2020;123:418-25.

11. Wada N, Kurokawa Y, Miyazaki Y, et al. The

Cite this article as: $\mathrm{Hu} \mathrm{C}$, Zhang $\mathrm{Y}, \mathrm{Xu} \mathrm{J}$, Chen $\mathrm{W}$, Yu P, Wang Y, Bao Z, Zhang R, Xu Z, Cheng X. Prognostic significance of serum tumor marker normalization in the perioperative period for patients with advanced gastric cancer. Ann Transl Med 2022;10(3):153. doi: 10.21037/atm-22-109 characteristics of the serum carcinoembryonic antigen and carbohydrate antigen 19-9 levels in gastric cancer cases. Surg Today 2017;47:227-32.

12. Toyoda H, Kumada T, Tada T, et al. Prognostic significance of a combination of pre- and post-treatment tumor markers for hepatocellular carcinoma curatively treated with hepatectomy. J Hepatol 2012;57:1251-7.

(English Language Editor: A. Kassem) 